\title{
Simultaneous Quenching for Semilinear Parabolic System with Localized Sources in a Square Domain
}

\author{
Wai Yuen Chan \\ Department of Mathematics, Texas A\&M University, Texarkana, TX, USA \\ Email: wychan@tamut.edu
}

How to cite this paper: Chan, W.Y. (2019) Simultaneous Quenching for Semilinear Parabolic System with Localized Sources in a Square Domain. Journal of Applied Mathematics and Physics, 7, 1473-1487. https://doi.org/10.4236/jamp.2019.77099

Received: May 30, 2019

Accepted: July 16, 2019

Published: July 19, 2019

Copyright $\odot 2019$ by author(s) and Scientific Research Publishing Inc. This work is licensed under the Creative Commons Attribution International License (CC BY 4.0).

http://creativecommons.org/licenses/by/4.0/

\section{cc) (i) Open Access}

\begin{abstract}
This paper studies quenching properties of solutions of a semilinear parabolic system with localized reaction sources in a square domain. The system has the homogeneous Dirichlet boundary condition and null initial condition. We prove that solutions quench simultaneously, and compute approximated critical values of the system using a numerical method.
\end{abstract}

\section{Keywords}

Simultaneous Quenching, Parabolic System, Conformal Mapping

\section{Introduction}

Let $\Gamma \in(0, \infty], a$ and $b$ be positive constants such that $a \leq b, \quad D=(-1,1) \times-(1,1)$, $\bar{D}=[-1,1] \times[-1,1]$,

$\partial D=\{\{-1\} \times[-1,1]\} \cup\{\{1\} \times[-1,1]\} \cup\{[-1,1] \times\{-1\}\} \cup\{[-1,1] \times\{1\}\}$. We also let $L$ be the operator such that $L u=u_{t}-\Delta u$. In this paper, we study the following semilinear parabolic system:

$$
\begin{aligned}
& L u=\frac{a}{1-v(0,0, t)} \text { in } D \times(0, \Gamma), \\
& L v=\frac{b}{1-u(0,0, t)} \text { in } D \times(0, \Gamma) \text {, } \\
& \left.\begin{array}{l}
u(x, y, 0)=0 \text { and } v(x, y, 0)=0 \text { on } \bar{D}, \\
u(x, y, t)=0 \text { and } v(x, y, t)=0 \text { on } \partial D \times(0, \Gamma) .
\end{array}\right\}
\end{aligned}
$$

Problem (1.1)-(1.2) illustrates the instabilities in some dynamical systems in 
which certain reactions are localized to electrodes, catalytic membranes, or other surfaces and local sites are immersed in a bulk medium happening at the origin $(0,0)$, see [1] [2]. Additionally, (1.1) describes a thermal ignition driven by the temperature at a single point, see [3]. Chadam, Peirce, and Yin [4] examined the blow-up set of solutions when the initial data are nontrivial and nonnegative bounded functions.

The quenching problem was initiated by Kawarada [5]. The model describes polarization phenomena in ionic conductors. Quenching also illustrates the phase transition between liquids and solids [6]. Chang, Hsu, and Liu [7] discussed the quenching rate of the problem (1.1)-(1.2) in an n-dimensional ball.

The solutions $u$ and $v$ are said to quench if there exists a finite time $\Gamma$ such that

$$
\begin{aligned}
& \max \{u(x, y, t):(x, y) \in \bar{D}\} \rightarrow 1^{-} \\
& \text {and } \max \{v(x, y, t):(x, y) \in \bar{D}\} \rightarrow 1^{-} \text {as } t \rightarrow \Gamma^{-} .
\end{aligned}
$$

To problem (1.1)-(1.2), there are critical values $a^{*}$ and $b^{\star}$ (both are positive) such that the maximum of solutions $u$ and $v$ reaches 1 in a finite time if $a>a^{*}$ and $b>b^{\star}$ while $u$ and $v$ exist globally and are bounded above by 1 if $a<a^{*}$ and $b<b^{\star}$, see [8].

The purposes of this paper are to prove solutions $u$ and $v$ to quench simultaneously at $(0,0)$, and use a numerical method to determine approximated values of $a^{*}$ and $b^{*}$ of the problem (1.1)-(1.2).

This paper is organized as follows. In Section 2, we prove that there are unique solutions $u$ and $v$ of the problem (1.1)-(1.2). In Section 3, we prove that either $u$ or $v$ quenches in a finite time. Then, we show that solutions $u$ and $v$ quench simultaneously at $(0,0)$. In Section 4 , we calculate approximated values of $a^{*}$ and $b^{*}$. This $a^{*}$ and $b^{*}$ associate with the existence of solutions of their steady state problem of the problem (1.1)-(1.2). Our numerical method is to evaluate an approximation of the steady solutions expressed in an integral representation form. For illustration, some examples are provided.

\section{Properties of $u$ and $v$}

Let $\beta_{1}(x, y, t)$ and $\beta_{2}(x, y, t)$ be nontrivial, nonnegative, and bounded functions on $\bar{D} \times(0, \infty)$. Here is a comparison theorem

Lemma 2.1. Suppose that $u(x, y, t)$ and $v(x, y, t)$ are solutions of the following system:

$$
\begin{aligned}
& L u \geq \beta_{1}(x, y, t) v(0,0, t) \text { in } D \times(0, \Gamma), \\
& L v \geq \beta_{2}(x, y, t) u(0,0, t) \text { in } D \times(0, \Gamma), \\
& u(x, y, 0) \geq 0 \text { and } v(x, y, 0) \geq 0 \text { on } \bar{D}, \\
& u(x, y, t) \geq 0 \text { and } v(x, y, t) \geq 0 \text { on } \partial D \times(0, \Gamma)
\end{aligned}
$$

Then, $u(x, y, t) \geq 0$ and $v(x, y, t) \geq 0$ on $\bar{D} \times[0, \Gamma)$.

Proof. Let $\varepsilon$ be a positive real number, and 


$$
\begin{aligned}
& \Phi(x, y, t)=u(x, y, t)+\varepsilon\left[\sin \left(\frac{\pi}{2}(x+1)\right)+\frac{1}{2}\right] \mathrm{e}^{\gamma t}, \\
& \Psi(x, y, t)=v(x, y, t)+\varepsilon\left[\sin \left(\frac{\pi}{2}(x+1)\right)+\frac{1}{2}\right] \mathrm{e}^{\gamma t},
\end{aligned}
$$

where $\gamma$ is a positive real number to be determined. From the construction, $\Phi(x, y, 0)>0$ and $\Psi(x, y, 0)>0$ on $\bar{D}$. By assumptions and a direction computation,

$$
\begin{aligned}
& \Phi_{t}-\Delta \Phi-\beta_{1} \Psi(0,0, t) \\
& =u_{t}-\Delta u+\varepsilon \mathrm{e}^{\gamma t}\left[\left(\gamma+\frac{\pi^{2}}{4}\right) \sin \left(\frac{\pi}{2}(x+1)\right)+\frac{\gamma}{2}\right]-\beta_{1}\left(v(0,0, t)+\frac{3 \varepsilon}{2} \mathrm{e}^{\gamma t}\right) \\
& \geq \frac{\varepsilon \mathrm{e}^{\gamma t}}{2}\left(\gamma-3 \beta_{1}\right) .
\end{aligned}
$$

We choose $\gamma$ such that $\gamma>3 \beta_{1}$. Thus,

$$
\Phi_{t}-\Delta \Phi-\beta_{1} \Psi(0,0, t)>0 \text { in } D \times(0, \Gamma) .
$$

Suppose $\Phi(x, y, t) \leq 0$ somewhere in $D \times(0, \Gamma)$. Then, the set

$$
\{t: \Phi(x, y, t) \leq 0 \text { for some }(x, y) \in D\}
$$

is non-empty. Let $\tilde{t}$ denote its infimum. Then, $0<\tilde{t}<\Gamma$ because $\Phi(x, y, 0)>0$ on $\bar{D}$. Thus, there exists some $\left(x_{1}, y_{1}\right) \in D$ such that $\Phi\left(x_{1}, y_{1}, \tilde{t}\right)=0$ and $\Phi_{t}\left(x_{1}, y_{1}, \tilde{t}\right) \leq 0$. On the other hand, $\Phi$ attains its local minimum at $\left(x_{1}, y_{1}, \tilde{t}\right)$. Therefore, $\Delta \Phi\left(x_{1}, y_{1}, \tilde{t}\right)>0$. Then, at $t=\tilde{t}$,

$$
\Phi_{t}\left(x_{1}, y_{1}, \tilde{t}\right)-\beta_{1} \Psi(0,0, \tilde{t})>L \Phi\left(x_{1}, y_{1}, \tilde{t}\right)-\beta_{1} \Psi(0,0, \tilde{t})>0 .
$$

Follow a similar argument, we assume that $\Psi(x, y, t) \leq 0$ somewhere in $D \times(0, \Gamma)$. Then, there exist some $\hat{t} \in(0, \Gamma)$ and $\left(x_{2}, y_{2}\right) \in D$ such that $\Psi\left(x_{2}, y_{2}, \hat{t}\right)=0, \quad \Psi_{t}\left(x_{2}, y_{2}, \hat{t}\right) \leq 0$, and $\Psi$ attains its local minimum at $\left(x_{2}, y_{2}, \hat{t}\right)$. Then at $t=\tilde{t}$

$$
\Psi_{t}\left(x_{2}, y_{2}, \hat{t}\right)-\beta_{2} \Phi(0,0, \hat{t})>L \Psi\left(x_{2}, y_{2}, \hat{t}\right)-\beta_{2} \Phi(0,0, \hat{t})>0 .
$$

Let us assume that $\hat{t}<\tilde{t}$. Since $\Phi$ attains its local minimum at $\left(x_{1}, y_{1}, \tilde{t}\right)$, $\Phi(0,0, \hat{t})>0$. By inequality $(2.2)$,

$$
0 \geq \Psi_{t}\left(x_{2}, y_{2}, \hat{t}\right) \geq \Psi_{t}\left(x_{2}, y_{2}, \hat{t}\right)-\beta_{2} \Phi(0,0, \hat{t})>0 .
$$

This gives a contradiction. Hence, $\Psi(x, y, t)>0$ in $D \times(0, \Gamma)$. Then, by (2.1), we show that $\Phi(x, y, t)>0$ in $D \times(0, \Gamma)$. Through a similar calculation, we obtain the same result when $\hat{t} \geq \tilde{t}$. Let $\varepsilon \rightarrow 0$, we have $u(x, y, t) \geq 0$ and $v(x, y, t) \geq 0$ in $D \times(0, \Gamma)$. Follow $u \geq 0$ and $v \geq 0$ on $\bar{D}$ and $\partial D \times(0, \Gamma)$, $u$ and $v$ are non-negative on $D \times[0, \Gamma)$. The proof is complete.

By Lemma 2.1, 0 is a lower solution of the problem (1.1)-(1.2). On the other hand, if $u$ and $v$ do not quench, then $\max _{\bar{D}} u<1$ and $\max _{\bar{D}} v<1$, and solutions $u$ and $v$ are bounded above by 1 . Further, $u$ and $v$ cease to exist when $u \geq 1$ and $v \geq 1$. Therefore, $0 \leq u<1$ and $0 \leq v<1$ on $\bar{D} \times[0, \Gamma)$. The exis- 
tence of classical solutions of the problem (1.1)-(1.2) is able to obtain by the Schauder fixed point theorem of [[9], pp. 502-504], and by Lemma $2.1 u$ and $v$ are unique.

Theorem 2.2. Problem (1.1)-(1.2) has unique classical solutions $u$ and $v \in C^{2+\alpha, 1+\alpha / 2}(\bar{D} \times[0, \Gamma))$ for some $\alpha \in(0,1)$ such that $0 \leq u, v<1$ on $\bar{D} \times[0, \Gamma)$.

Lemma 2.3. $u_{t} \geq 0$ and $v_{t} \geq 0$ on $\bar{D} \times[0, \Gamma)$. Further, $u_{t}>0$ and $v_{t}>0$ in $D \times(0, \Gamma)$.

Proof. From Theorem 2.2, $u(x, y, t)$ and $v(x, y, t)$ are nonnegative on $\bar{D} \times[0, \Gamma)$. Let $h$ be a real number in $(0, \Gamma)$. Then, $u(x, y, h) \geq 0$ and $v(x, y, h) \geq 0$ on $\bar{D}$, and $u(x, y, t+h)=0$ and $v(x, y, t+h)=0$ on $\partial D \times[0, \Gamma-h)$. By the mean value theorem, there exist $u_{1}$ (between $u(0,0, t+h)$ and $\left.u(0,0, t)\right)$ and $v_{1}$ (between $v(0,0, t+h)$ and $\left.v(0,0, t)\right)$ such that

$$
\begin{aligned}
& L u(x, y, t+h)-L u(x, y, t)=\frac{a}{\left(1-v_{1}\right)^{2}}(v(0,0, t+h)-v(0,0, t)), \\
& L v(x, y, t+h)-L v(x, y, t)=\frac{b}{\left(1-u_{1}\right)^{2}}(u(0,0, t+h)-u(0,0, t)) .
\end{aligned}
$$

By Lemma 2.1, $u(x, y, t+h) \geq u(x, y, t)$ and $v(x, y, t+h) \geq v(x, y, t)$ on $\bar{D} \times[0, \Gamma-h)$. This gives

$\frac{u(x, y, t+h)-u(x, y, t)}{h} \geq 0$ and $\frac{v(x, y, t+h)-v(x, y, t)}{h} \geq 0$ on $\bar{D} \times[0, \Gamma-h)$.

Taking $h \rightarrow 0, u_{t}$ and $v_{t}$ are nonnegative on $\bar{D} \times[0, \Gamma)$, respectively. To show that $u_{t}$ and $v_{t}$ are positive, let us differentiate (1.1) with respect to $t$. Then, $u_{t}$ and $V_{t}$ satisfy

$$
\begin{aligned}
& L u_{t}=\frac{a}{(1-v(0,0, t))^{2}} v_{t}(0,0, t) \geq 0 \text { in } D \times(0, \Gamma), \\
& L v_{t}=\frac{b}{(1-u(0,0, t))^{2}} u_{t}(0,0, t) \geq 0 \text { in } D \times(0, \Gamma), \\
& \left.\begin{array}{l}
u_{t}(x, y, 0) \geq 0 \text { and } v_{t}(x, y, 0) \geq 0 \text { on } \bar{D}, \\
u_{t}(x, y, t)=0 \text { and } v_{t}(x, y, t)=0 \text { on } \partial D \times(0, \Gamma) .
\end{array}\right\}
\end{aligned}
$$

By the maximum principle [[10], p. 54], $u_{t}>0$ and $v_{t}>0$ in $D \times(0, \Gamma)$.

\section{Simultaneous Quenching and Global Existence}

In this section, we show that either $u$ or $v$ quenches in a finite time first. Then, we prove that $u$ and $v$ quench in the same time at $(0,0)$. Afterward, we prove that the problem (1.1)-(1.2) has a global solution when $a$ and $b$ are sufficiently small.

Lemma 3.1. $u$ and $v$ both attain their maximum at $(0,0)$ for all $t \in(0, \Gamma)$.

Proof. It suffices to prove that $u$ attains its maximum along the $x$ and $y$ axes. Let us consider the first equation of (1.1) along the $x$-axis, we have

$$
L u(x, 0, t)=u_{t}(x, 0, t)-u_{x x}(x, 0, t)=\frac{a}{1-v(0,0, t)} .
$$


Differentiate the above equation with respect to $x$ to yield

$$
L u_{x}(x, 0, t)=0 \text {. }
$$

By the symmetry of $D$ with respect to the $x$ and $y$ axes, $u_{x}(0,0, t)=0$ for all $t \in(0, \Gamma)$. By the Hopf's lemma [11], p. 170], $u_{x}(-1,0, t)>0$ for all $t \in(0, \Gamma)$. At $t=0, u_{x}(x, 0,0)=0$ for all $x \in[-1,0]$. By the maximum principle, $u_{x}(x, 0, t)>0$ for all $x \in[-1,0)$ when $t \in(0, \Gamma)$. Similarly, for all $t \in(0, \Gamma)$, we obtain $u_{x}(x, 0, t)<0$ when $x \in(0,1]$. Therefore, $u(0,0, t) \geq u(x, 0, t)$ for all $x \in[-1,1]$ when $t \in(0, \Gamma)$. Likewise, $u(0,0, t) \geq u(0, y, t)$ for all $y \in[-1,1]$ when $t \in(0, \Gamma)$. Thus, $u(0,0, t) \geq u(x, y, t)$ on $\bar{D} \times(0, \Gamma)$. Similarly, $v(0,0, t) \geq v(x, y, t)$ on $\bar{D} \times(0, \Gamma)$. Hence, $u$ and $v$ both attain their maximum at $(0,0)$ for all $t \in(0, \Gamma)$.

Let $\phi_{1}$ be the eigenfunction corresponding to the first eigenvalue $\lambda_{1}(>0)$ of the Sturm-Liuoville problem below,

$$
\Delta \phi+\lambda \phi=0 \text { in } D, \phi=0 \text { on } \partial D .
$$

This eigenfunction has the properties: $\phi_{1}>0$ in $D$ and $\iint \phi_{1} \mathrm{~d} x \mathrm{~d} y=1$ [[12], p. $10]$. Let $c$ be a positive real number such that $c \leq a b /\left(a+b^{D}\right)$. We show that either $u$ or $v$ quenches in a finite time.

Lemma 3.2. If $2 \sqrt{2} c>\lambda_{1}$, where $c \leq a b /(a+b)$, then either $u$ or $v$ quenches on $\bar{D}$ in a finite time $\tilde{\Gamma}$.

Proof. By Lemma 3.1, $u(0,0, t) \geq u(x, y, t)$ and $v(0,0, t) \geq v(x, y, t)$ on $\bar{D} \times(0, \Gamma)$. Let $\hat{u}(x, y, t)$ and $\hat{v}(x, y, t)$ be the solutions to the following parabolic system:

$$
\left.\begin{array}{c}
L \hat{u}=\frac{a}{1-\hat{v}(x, y, t)} \text { in } D \times(0, \Gamma), \\
L \hat{v}=\frac{b}{1-\hat{u}(x, y, t)} \text { in } D \times(0, \Gamma), \\
\hat{u}(x, y, 0)=0 \text { and } \hat{v}(x, y, 0)=0 \text { on } \bar{D}, \\
\hat{u}(x, y, t)=0 \text { and } \hat{v}(x, y, t)=0 \text { on } \partial D \times(0, \Gamma) .
\end{array}\right\}
$$

By the maximum principle, $\hat{u}(x, y, t) \geq 0$ and $\hat{v}(x, y, t) \geq 0$ on $\bar{D} \times[0, \Gamma)$. Further, $u-\hat{u}$ and $v-\hat{v}$ satisfy the system below:

$$
\begin{aligned}
& L(u-\hat{u})=\frac{a}{1-v(0,0, t)}-\frac{a}{1-\hat{v}(x, y, t)} \geq \frac{a}{1-v(x, y, t)}-\frac{a}{1-\hat{v}(x, y, t)}, \\
& L(v-\hat{v})=\frac{b}{1-u(0,0, t)}-\frac{b}{1-\hat{u}(x, y, t)} \geq \frac{b}{1-u(x, y, t)}-\frac{b}{1-\hat{u}(x, y, t)} .
\end{aligned}
$$

By $u-\hat{u}=0$ and $v-\hat{v}=0$ on $\bar{D}$ and $\partial D \times(0, \Gamma)$, and the maximum principle, we have $u \geq \hat{u}$ and $v \geq \hat{v}$ on $\bar{D} \times[0, \Gamma)$. It suffices to prove either $\hat{u}$ or $\hat{v}$ to quench over $\bar{D}$ in a finite time.

Multiplying $\phi_{1}$ on both sides of (3.1) and integrating expressions over the domain $D$, we obtain 


$$
\begin{aligned}
& \iint_{D} \hat{u}_{t} \phi_{1} \mathrm{~d} x \mathrm{~d} y-\iint_{D} \Delta \hat{u} \phi_{1} \mathrm{~d} x \mathrm{~d} y=a \iint_{D} \frac{\phi_{1}}{1-\hat{v}} \mathrm{~d} x \mathrm{~d} y, \\
& \iint_{D} \hat{v}_{t} \phi_{1} \mathrm{~d} x \mathrm{~d} y-\iint_{D} \Delta \hat{v} \phi_{1} \mathrm{~d} x \mathrm{~d} y=b \iint_{D} \frac{\phi_{1}}{1-\hat{u}} \mathrm{~d} x \mathrm{~d} y .
\end{aligned}
$$

Use the Green's second identity [[10], p. 96] and (3.2), it yields

$$
\begin{aligned}
& \left(\iint_{D} \hat{u} \phi_{1} \mathrm{~d} x \mathrm{~d} y\right)_{t}=-\lambda_{1} \iint_{D} \hat{u} \phi_{1} \mathrm{~d} x \mathrm{~d} y+a \iint_{D} \frac{\phi_{1}}{1-\hat{v}} \mathrm{~d} x \mathrm{~d} y, \\
& \left(\iint_{D} \hat{v} \phi_{1} \mathrm{~d} x \mathrm{~d} y\right)_{t}=-\lambda_{1} \iint_{D} \hat{v} \phi_{1} \mathrm{~d} x \mathrm{~d} y+b \iint_{D} \frac{\phi_{1}}{1-\hat{u}} \mathrm{~d} x \mathrm{~d} y .
\end{aligned}
$$

By the Maclaurin's series, we have

$$
\begin{aligned}
& \left(\iint_{D} \hat{u} \phi_{1} \mathrm{~d} x \mathrm{~d} y\right)_{t} \geq-\lambda_{1} \iint_{D} \hat{u} \phi_{1} \mathrm{~d} x \mathrm{~d} y+a \iint_{D} \hat{v}^{2} \phi_{1} \mathrm{~d} x \mathrm{~d} y+a \iint_{D} \phi_{1} \mathrm{~d} x \mathrm{~d} y, \\
& \left(\iint_{D} \hat{v} \phi_{1} \mathrm{~d} x \mathrm{~d} y\right)_{t} \geq-\lambda_{1} \iint_{D} \hat{v} \phi_{1} \mathrm{~d} x \mathrm{~d} y+b \iint_{D} \hat{u}^{2} \phi_{1} \mathrm{~d} x \mathrm{~d} y+b \iint_{D} \phi_{1} \mathrm{~d} x \mathrm{~d} y .
\end{aligned}
$$

Let $R(t)=\iint_{D} \hat{u} \phi_{1} \mathrm{~d} x \mathrm{~d} y$ and $P(t)=\iint_{D} \hat{v} \phi_{1} \mathrm{~d} x \mathrm{~d} y$. Adding above inequalities together and using the Jensen's inequality [[12], p. 11], we obtain

$$
\frac{\mathrm{d}}{\mathrm{d} t}(R+P) \geq-\lambda_{1}(R+P)+a P^{2}+b R^{2}+a+b .
$$

As $c \leq a b /(a+b) \leq a \leq b$, we have

$$
\frac{(a-c) P^{2}+(b-c) R^{2}}{2} \geq \sqrt{(a+b)\left(\frac{a b}{a+b}-c\right)+c^{2}} P R \geq c P R .
$$

Hence,

$$
a P^{2}+b R^{2} \geq c(R+P)^{2}
$$

Then, differential inequality (3.3) becomes

$$
\frac{\mathrm{d}}{\mathrm{d} t}(R+P) \geq-\lambda_{1}(R+P)+c(R+P)^{2}+2 c .
$$

Let $E(t)=R(t)+P(t)$. Then, $E(t) \geq 0$ in $[0, T)$ and

$$
\frac{\mathrm{d}}{\mathrm{d} t} E \geq-\lambda_{1} E+c E^{2}+2 c
$$

Using separation of variables and integrating both sides over $(0, t)$, we obtain

$$
t \leq \frac{2}{\sqrt{8 c^{2}-\lambda_{1}^{2}}}\left[\tan ^{-1}\left(\frac{2 c E(t)-\lambda_{1}}{\sqrt{8 c^{2}-\lambda_{1}^{2}}}\right)+\tan ^{-1}\left(\frac{\lambda_{1}}{\sqrt{8 c^{2}-\lambda_{1}^{2}}}\right)\right] .
$$

Suppose that $E(t)$ exists for all $t>0$. By the assumption $2 \sqrt{2} c>\lambda_{1}$, we have

$$
\tan ^{-1}\left[\left(2 c E(t)-\lambda_{1}\right) / \sqrt{8 c^{2}-\lambda_{1}^{2}}\right] \rightarrow \infty \text { if } t \rightarrow \infty .
$$

But, $\tan ^{-1}\left[\left(2 c E(t)-\lambda_{1}\right) / \sqrt{8 c^{2}-\lambda_{1}^{2}}\right]$ is bounded above by $\pi / 2$. This is a 
contradiction. It implies that $E(t)$ ceases to exist in a finite time $\hat{\Gamma}$. This shows that either $R(t)$ or $P(t)$ does not exist when $t$ approaches $\hat{\Gamma}$. Thus, either $\hat{u}$ or $\hat{v}$ quenches on $\bar{D}$ at $\hat{\Gamma}$. Since $u \geq \hat{u}$ and $v \geq \hat{v}$, either $u$ or $v$ quenches on $\bar{D}$ in a finite time $\tilde{\Gamma}$ where $\tilde{\Gamma} \leq \hat{\Gamma}$.

From the result of Lemma 3.1, we know that $(0,0)$ is a quenching point of $u$ and $V$ if they quench. Let $\Gamma^{*}$ be the supremum of the time $\Gamma$ for which the problem (1.1)-(1.2) has unique solutions $u$ and $v$.

Theorem 3.3. If $\Gamma^{*}<\infty$, then either $u(0,0, t)$ or $v(0,0, t)$ quenches at $\Gamma^{*}$.

Proof. Suppose that both $u$ and $v$ do not quench at $(0,0)$ when $t=\Gamma^{*}$. Then, there exist positive constants $k_{1}$ and $k_{2}$ such that $u(0,0, t) \leq k_{1}<1$ and $v(0,0, t) \leq k_{2}<1$ for all $t \in\left[0, \Gamma^{*}\right]$. This shows that $a /(1-v(0,0, t))<Q_{1}$ and $b /(1-u(0,0, t))<Q_{2}$ for some positive constants $Q_{1}$ and $Q_{2}$ when $t \in\left[0, \Gamma^{*}\right]$. Then, by Theorem 4.2.1 of [[13], p. 139], $u$ and $v \in C^{2+\alpha, 1+\alpha / 2}\left(\bar{D} \times\left[0, \Gamma^{*}\right]\right)$. This implies that there exist positive constants $k_{3}$ and $k_{4}$ such that $u(x, y, t) \leq k_{3}<1$ and $v(x, y, t) \leq k_{4}<1$ for all $(x, y, t) \in \bar{D} \times\left[0, \Gamma^{*}\right]$. In order to arrive a contradiction, we need to show that $u$ and $v$ can continue to exist in a larger time interval $\left[0, \Gamma^{*}+t_{1}\right)$ for some positive $t_{1}$. This can be achieved by extending the upper bound. Let us construct upper solutions $\psi(x, y, t)=k_{3} f(t)$ and $\sigma(x, y, t)=k_{4} g(t)$, where $f(t)$ and $g(t)$ are solutions of the following differential system:

$$
\begin{aligned}
& \frac{\mathrm{d}}{\mathrm{d} t} k_{3} f(t)=\frac{a}{1-k_{4} g(t)} \text { for } t>\Gamma^{*}, f\left(\Gamma^{*}\right)=1, \\
& \frac{\mathrm{d}}{\mathrm{d} t} k_{4} g(t)=\frac{b}{1-k_{3} f(t)} \text { for } t>\Gamma^{*}, g\left(\Gamma^{*}\right)=1 .
\end{aligned}
$$

By $k_{3} f\left(\Gamma^{*}\right)<1, k_{4} g\left(\Gamma^{*}\right)<1$, and the Picard iteration, $f(t)$ and $g(t)$ are positive functions, and $a /\left[1-k_{4} g(t)\right]>0$ and $b /\left[1-k_{3} f(t)\right]>0$. This implies that $f(t)$ and $g(t)$ are increasing functions of $t$. Let $t_{1}$ be a positive real number determined by $k_{3} f\left(\Gamma^{*}+t_{1}\right)=k_{5}<1$ and $k_{4} g\left(\Gamma^{*}+t_{1}\right)=k_{6}<1$ for some positive constants $k_{5}$ and $k_{6}$ greater than $k_{3}$ and $k_{4}$ respectively. By our construction, $\psi(x, y, t)=\psi(0,0, t)$ and $\sigma(x, y, t)=\sigma(0,0, t)$ satisfy,

$$
\begin{gathered}
L \psi(x, y, t)=\frac{a}{1-\sigma(0,0, t)} \text { in } D \times\left(\Gamma^{*}, \Gamma^{*}+t_{1}\right), \\
L \sigma(x, y, t)=\frac{b}{1-\psi(0,0, t)} \text { in } D \times\left(\Gamma^{*}, \Gamma^{*}+t_{1}\right), \\
\psi\left(x, y, \Gamma^{*}\right)=k_{3} \geq u\left(x, y, \Gamma^{*}\right) \text { and } \sigma\left(x, y, \Gamma^{*}\right)=k_{4} \geq v\left(x, y, \Gamma^{*}\right) \text { on } \bar{D}, \\
\psi(x, y, t)=k_{3} f(t)>0 \text { and } \sigma(x, y, t)=k_{4} g(t)>0 \text { on } \partial D \times\left[\Gamma^{*}, \Gamma^{*}+t_{1}\right) .
\end{gathered}
$$

By Lemma 2.1, $\psi(x, y, t) \geq u(x, y, t)$ and $\sigma(x, y, t) \geq v(x, y, t)$ on $\bar{D} \times\left[\Gamma^{*}, \Gamma^{*}+t_{1}\right)$. Therefore, we find solutions $u$ and $v$ to the problems (1.1)-(1.2) on $\bar{D} \times\left[\Gamma^{*}, \Gamma^{*}+t_{1}\right)$. This contradicts the definition of $\Gamma^{*}$. Hence, either $u(0,0, t)$ or $v(0,0, t)$ quenches at $\Gamma^{*}$. 
Let $\varphi_{0}(x, y) \in C(\bar{D}) \cap C^{2}(D)$ such that $\Delta \varphi_{0}(x, y)<0, \varphi_{0}(x, y)>0$ in $D$, and $\varphi_{0}(x, y)=0$ on $\partial D$ and $\max _{x \in \bar{D}} \varphi_{0}(x, y) \leq 1$. Let $\varphi(x, y, t)$ be the solution to the following first initial-boundary value problem:

$$
\begin{aligned}
& L w=0 \text { in } D \times(0, \infty), \\
& w(x, y, 0)=\varphi_{0}(x, y) \text { on } \bar{D}, \\
& w(x, y, t)=0 \text { on } \partial D \times(0, \infty) .
\end{aligned}
$$

By the maximum principle, $\varphi(x, y, t)>0$ in $D \times[0, \infty)$ and is bounded above by $\varphi_{0}(x, y)$, and it satisfies

$$
\max _{(x, y, t) \in \bar{D} \times[0, \infty)} \varphi(x, y, t) \leq 1 .
$$

Let $t_{2} \in(0, \Gamma)$ such that $v\left(0,0, t_{2}\right) \leq k_{7}<1$ for some positive constant $k_{7}$. Then,

$$
\frac{a \varphi\left(x, y, t_{2}\right)}{1-k_{7}} \geq \frac{a \varphi\left(x, y, t_{2}\right)}{1-v\left(0,0, t_{2}\right)} .
$$

As $u_{t}\left(x, y, t_{2}\right)>0$ and $\varphi\left(x, y, t_{2}\right)>0$ in $D$, and $u_{t}\left(x, y, t_{2}\right)=\varphi\left(x, y, t_{2}\right)=0$ on $\partial D$, we choose a positive real number $\mu_{1}$ less than 1 such that

$$
u_{t}\left(x, y, t_{2}\right) \geq \frac{\mu_{1} a \varphi\left(x, y, t_{2}\right)}{1-k_{7}} \text { on } \bar{D} \text {. }
$$

Also, $u_{t}(x, y, t)=\mu_{1} a \varphi(x, y, t) /[1-v(0,0, t)]$ for all $(x, y, t) \in \partial D \times[0, \Gamma)$. Let us define $I(x, y, t)=u_{t}(x, y, t)-\mu_{1} a \varphi(x, y, t) /[1-v(0,0, t)]$. By inequalities (3.4) and (3.5), $I\left(x, y, t_{2}\right) \geq 0$ on $\bar{D}$. Let

$H(x, y, t)=v_{t}(x, y, t)-\mu_{2} b \varphi(x, y, t) /[1-u(0,0, t)]$ where $\mu_{2}$ is a positive real number less than 1 . Similar to the previous argument, we choose $\mu_{2}$ such that $H\left(x, y, t_{2}\right) \geq 0$ on $\bar{D}$. We modify the proof of Lemma 3.4 of [7] to obtain the result below.

Lemma 3.4. $I(x, y, t) \geq 0$ and $H(x, y, t) \geq 0$ on $\bar{D} \times\left[t_{2}, \Gamma\right)$.

Proof. By a direct computation,

$$
\begin{aligned}
& I_{t}=u_{t t}-\frac{\mu_{1} a \varphi}{[1-v(0,0, t)]^{2}} v_{t}(0,0, t)-\frac{\mu_{1} a \varphi_{t}}{1-v(0,0, t)}, \\
& \Delta I=\Delta u_{t}-\frac{\mu_{1} a}{1-v(0,0, t)} \Delta \varphi .
\end{aligned}
$$

From the above expression, we have

$$
L I=\frac{a}{[1-v(0,0, t)]^{2}} v_{t}(0,0, t)\left(1-\mu_{1} \varphi\right) \text { in } D \times(0, \Gamma) .
$$

By $\varphi \leq 1$ on $\bar{D} \times[0, \infty), \mu_{1}<1$, and $v_{t}(0,0, t)>0$ for all $t \in(0, \Gamma)$, it gives $L I \geq 0$ in $D \times(0, \Gamma)$. In addition, $I\left(x, y, t_{2}\right) \geq 0$ on $\bar{D}$, and $I(x, y, t)=0$ on $\partial D \times\left(t_{2}, \Gamma\right)$. By the maximum principle, $I(x, y, t) \geq 0$ on $\bar{D} \times\left[t_{2}, \Gamma\right)$. Similarly, $H(x, y, t) \geq 0$ on $\bar{D} \times\left[t_{2}, \Gamma\right)$.

Here is the result of simultaneous quenching.

Theorem 3.5. If either $u$ or $v$ quenches at $(0,0)$ when $t=\Gamma$, then $u$ and $v$ 
quench simultaneously at $(0,0)$ when $t=\Gamma$.

Proof. If not, let us assume that $v$ quenches at $(0,0)$ when $t=\Gamma$ but $u$ continues to exist beyond $\Gamma$. That is, there exists a positive constant $k_{8}$ such that $0 \leq u \leq k_{8}<1$ for all $(x, y, t) \in \bar{D} \times\left[0, \Gamma+t_{3}\right)$ for some $t_{3}>0$. By Lemma 3.4, we have

$$
u_{t}(0,0, t) \geq \frac{\mu_{1} a \varphi(0,0, t)}{1-v(0,0, t)} \text { and } v_{t}(0,0, t) \geq \frac{\mu_{2} b \varphi(0,0, t)}{1-u(0,0, t)} \text { for all } t \in\left[t_{2}, \Gamma\right) .
$$

By Lemma 3.1, $u$ and $v$ both attain their maximum at $(0,0)$ for all $t \in(0, \Gamma)$. Then, $\Delta u(0,0, t)<0$ and $\Delta v(0,0, t)<0$ on $\left[t_{2}, \Gamma\right)$. Combine (1.1) with above inequalities to give

$$
\begin{aligned}
& \frac{\mu_{1} a \varphi(0,0, t)}{1-v(0,0, t)} \leq u_{t}(0,0, t)<\frac{a}{1-v(0,0, t)} \\
& \frac{\mu_{2} b \varphi(0,0, t)}{1-u(0,0, t)} \leq v_{t}(0,0, t)<\frac{b}{1-u(0,0, t)}
\end{aligned}
$$

From them, we get a compound inequality

$$
\frac{\mu_{1} a \varphi(0,0, t)}{1-v(0,0, t)} \frac{1-u(0,0, t)}{b} \leq \frac{\mathrm{d} u(0,0, t)}{\mathrm{d} v(0,0, t)} \leq \frac{a}{1-v(0,0, t)} \frac{1-u(0,0, t)}{\mu_{2} b \varphi(0,0, t)} .
$$

From the left-side inequality, we have

$$
\frac{\mu_{1} a \varphi(0,0, t)}{b[1-v(0,0, t)]} \mathrm{d} v(0,0, t) \leq \frac{\mathrm{d} u(0,0, t)}{1-u(0,0, t)}
$$

Since $0<\varphi(0,0, t) \leq 1$ for all $t \in(0, \infty)$, there exists a positive constant $\delta>0$ such that $\varphi(0,0, t)>\delta$ for all $t \in\left[t_{2}, \Gamma\right)$. Integrating both sides over the interval $\left[t_{2}, s\right]$ where $s \in\left(t_{2}, \Gamma\right)$, we obtain

$$
\begin{aligned}
& \frac{\mu_{1} a \delta}{b}\left\{\ln [1-v(0,0, s)]-\ln \left[1-v\left(0,0, t_{2}\right)\right]\right\} \\
& \geq \ln [1-u(0,0, s)]-\ln \left[1-u\left(0,0, t_{2}\right)\right] .
\end{aligned}
$$

By assumption, $v$ quenches at $(0,0)$ when $t=\Gamma$, we have $\ln [1-v(0,0, s)] \rightarrow-\infty$ as $s \rightarrow \Gamma^{-}$. Since $\ln \left[1-v\left(0,0, t_{2}\right)\right]$ and $\ln \left[1-u\left(0,0, t_{2}\right)\right]$ are both bounded, the above inequality implies $\ln [1-u(0,0, s)] \rightarrow-\infty$ as $s \rightarrow \Gamma^{-}$. Therefore, $u$ quenches at $(0,0)$ when $t=\Gamma$. It contradicts that $u$ exists on $\bar{D} \times\left[0, \Gamma+t_{3}\right)$. Follow the second half of inequality $(3.5)$, we can prove that $v$ quenches at $(0,0)$ when $t=\Gamma$ if $u$ quenches at $(0,0)$ when $t=\Gamma$. The proof is complete.

Now, we prove that $u$ and $v$ exist globally when $a$ and $b$ are sufficiently small. Our method is to construct global-exist upper solutions of the problem (1.1)-(1.2).

Lemma 3.6. If $a$ and $b$ are sufficiently small, then there is a global solution to the problem (1.1)-(1.2).

Proof. It suffices to construct upper solutions which exist all time. Let $q(x, y)=A\left(2-x^{2}-y^{2}\right)$ and $m(x, y)=B\left(2-x^{2}-y^{2}\right)$ where $A$ and $B$ are positive real numbers such that $A<1 / 2$ and $B<1 / 2$. Clearly, $0 \leq q, m<1$ for 
all $(x, y) \in \bar{D}$. In addition,

$$
L q-\frac{a}{1-m(0,0)}=4 A-\frac{a}{1-2 B} .
$$

If $a$ is sufficiently small, then we have the inequality: $4 A \geq a /(1-2 B)$. This leads to

$$
L q \geq \frac{a}{1-m(0,0)} \text { in } D \times(0, \infty) .
$$

Similarly, if $b$ is sufficiently small, we have $4 B \geq b /(1-2 A)$ and

$$
L m \geq \frac{b}{1-q(0,0)} \text { in } D \times(0, \infty) .
$$

By Lemma 2.1, $q(x, y) \geq u(x, y, t)$ and $m(x, y) \geq v(x, y, t)$ on $\bar{D} \times[0, \infty)$. Hence, $u$ and $v$ both exist globally. The proof is complete.

Lemma 3.7. $u$ and $v$ are non-decreasing functions in $a$ and $b$ respectively.

Proof. Let $\tilde{u}$ and $\tilde{v}$ be solutions to the problem (1.1)-(1.2) corresponding to $a=a_{1}$ and $b=b_{1}$, and $\bar{u}$ and $\bar{v}$ be solutions when $a=a_{2}$ and $b=b_{2}$, where $a_{1} \geq a_{2}$ and $b_{1} \geq b_{2}$. Then, $\tilde{u}-\bar{u}$ and $\tilde{v}-\bar{v}$ satisfy the parabolic system:

$$
\begin{aligned}
& L(\tilde{u}-\bar{u})=\frac{a_{1}}{1-\tilde{v}(0,0, t)}-\frac{a_{2}}{1-\bar{v}(0,0, t)} \geq \frac{a_{2}}{1-\tilde{v}(0,0, t)}-\frac{a_{2}}{1-\bar{v}(0,0, t)}, \\
& L(\tilde{v}-\bar{v})=\frac{b_{1}}{1-\tilde{u}(0,0, t)}-\frac{b_{2}}{1-\bar{u}(0,0, t)} \geq \frac{b_{2}}{1-\tilde{u}(0,0, t)}-\frac{b_{2}}{1-\bar{u}(0,0, t)} .
\end{aligned}
$$

As $\tilde{u}-\bar{u}=0$ and $\tilde{v}-\bar{v}=0$ on $\bar{D}$ and $\partial D \times(0, \Gamma)$, we have $\tilde{u} \geq \bar{u}$ and $\tilde{v} \geq \bar{v}$ by Lemma 2.1.

\section{Approximated Values of $a^{*}$ and $b^{*}$}

Let $U(x, y)$ and $V(x, y)$ be steady-state solutions of the problem (1.1)-(1.2). They satisfy

$$
\begin{aligned}
& -\Delta U=\frac{a}{1-V(0,0)} \text { in } D, \\
& -\Delta V=\frac{b}{1-U(0,0)} \text { in } D \text {, } \\
& U(x, y)=0 \text { and } V(x, y)=0 \text { on } \partial D \text {. }
\end{aligned}
$$

From Lemma 2.3, $u_{t}(x, y, t) \geq 0$ and $v_{t}(x, y, t) \geq 0$ on $\bar{D}$ for all $t \geq 0$. Based on Theorem 10.4.2 of [[10], pp. 532-533], we have the following result.

Lemma 4.1. If $0 \leq u(x, y, t)<1$ and $0 \leq v(x, y, t)<1$ on $\bar{D} \times[0, \infty)$, then $u$ and $v$ converge monotonically to $U(x, y)$ and $V(x, y)$ on $\bar{D}$ respectively as $t \rightarrow \infty$.

Let $G(x, y ; \xi, \eta)$ be the Green's function of the operator: $-\Delta$ over the domain $D$. The integral representation of the solution of the problem (4.1)-(4.2) is given by 


$$
\begin{aligned}
& U(x, y)=\iint_{D} \frac{a}{1-V(0,0)} G(x, y ; \xi, \eta) \mathrm{d} \xi \mathrm{d} \eta, \\
& V(x, y)=\iint_{D} \frac{b}{1-U(0,0)} G(x, y ; \xi, \eta) \mathrm{d} \xi \mathrm{d} \eta .
\end{aligned}
$$

By Lemma 3.7, $u$ and $v$ are respectively non-decreasing functions in $a$ and $b$. Then, by Lemma 3.6, there exist $a^{*}$ and $b^{*}$ for which $u$ and $v$ exist globally and less than 1 if $a<a^{*}$ and $b<b^{*}$. By Lemma 4.1, $u$ and $v$ converge to $U$ and $V$ when $a<a^{*}$ and $b<b^{*}$. Thus, $U$ and $V$ exist and they are bounded above by 1 when $a<a^{*}$ and $b<b^{*}$, and $\max _{\bar{D}} U(x, y)=1$ and $\max _{\bar{D}} V(x, y)=1$ if $a>a^{*}$ and $b>b^{*}$.

Let us construct sequences of integral solutions: $\left\{U_{j}(x, y)\right\}_{j=1}^{\infty}$ and $\left\{V_{j}(x, y)\right\}_{j=1}^{\infty}$ such that $U_{0}(x, y) \equiv 0, V_{0}(x, y) \equiv 0$, and they satisfy

$$
\begin{aligned}
& U_{j}(x, y)=\iint_{D} \frac{a}{1-V_{j-1}(0,0)} G(x, y ; \xi, \eta) \mathrm{d} \xi \mathrm{d} \eta, \\
& V_{j}(x, y)=\iint_{D} \frac{b}{1-U_{j-1}(0,0)} G(x, y ; \xi, \eta) \mathrm{d} \xi \mathrm{d} \eta,
\end{aligned}
$$

for $j=1,2,3, \cdots$. We follow Theorem 4 of [14] to obtain the following result.

Theorem 4.2. Suppose that $a<a^{*}$ and $b<b^{*}$, the sequences $\left\{U_{j}\right\}_{j=1}^{\infty}$ and $\left\{V_{j}\right\}_{j=1}^{\infty}$ converge monotonically to solutions $U$ and $V$ of the Equations (4.3) and (4.4) where $0<U_{j-1}<U_{j}<U<1$ and $0<V_{j-1}<V_{j}<V<1$ in $D$ for $j=2,3, \cdots$.

To determine $G(x, y ; \xi, \eta)$, we map the domain $D$ onto the unit disk $S$ : $\sqrt{x^{2}+y^{2}}<1$ through a conformal mapping. Let $J$ denote this mapping. By the Riemann Mapping Theorem, $J$ exists and is unique. This theorem is stated below.

Theorem 4.3 (Riemann Mapping Theorem). Suppose that $z$ is a point locating in $\Lambda$ which is a simply-connected two-dimensional domain with more than one boundary point, and $v$ is a point of $\Lambda$, then there exists a unique analytic function $\varsigma=J(z)$ which is regular in $\Lambda$ and maps $\Lambda$ conformally onto the unit disk $S:|\varsigma|<1$ in such a way that $J(v)=0$ and $J^{\prime}(v)>0$.

Let $z=x+i y$ and $\tilde{z}=\xi+i \eta$ be some points in a simply-connected two dimensional domain $\Lambda$. From the result of [[15], pp. 288 and 304], the Green's function is positive in $\Lambda$ and is given by

$$
G(x, y ; \xi, \eta)=\frac{1}{2 \pi} \ln \left|\frac{1-\overline{J(\tilde{z})} J(z)}{J(z)-J(\tilde{z})}\right|,
$$

where $J(z)=(z-\tilde{z}) \mathrm{e}^{-2 \pi p(z)}$, and $p(z)$ is a real harmonic function in $\Lambda$. With this $J(z)$, we map $\Lambda$ onto $S$ conformally. (4.5) is expressed as

$$
G(x, y ; \xi, \eta)=p(z)-\frac{1}{2 \pi} \ln |z-\tilde{z}| .
$$

The Taylor series representation of $p(z)$ with respect to $\tilde{z}$ is given by 


$$
p(z)=\sum_{j=0}^{\infty} \hat{c}_{j}(z-\tilde{z})^{j},
$$

where $\hat{c}_{j}$ is a complex number given by $\hat{c}_{j}=\hat{a}_{j}+i \hat{b}_{j}$. Let $z-\tilde{z}=r \mathrm{e}^{i \theta}$ where $r=|z-\tilde{z}|$ and $\theta$ is the angle between the line segment $z \tilde{z}$ and the positive $x$-axis. Then, the above series is represented by

$$
p(z)=\sum_{j=0}^{\infty} r^{j}\left[\left(\hat{a}_{j} \cos j \theta-\hat{b}_{j} \sin j \theta\right)+i\left(\hat{b}_{j} \cos j \theta+\hat{a}_{j} \sin j \theta\right)\right] .
$$

To determine an approximation of $G(x, y ; \xi, \eta)$, we let $\Lambda=D$. By $p(z)$ is a real function, we have

$$
p(z)=\sum_{j=0}^{\infty} r^{j}\left(\hat{a}_{j} \cos j \theta-\hat{b}_{j} \sin j \theta\right) .
$$

From the symmetry of $D$ with respect to the $x$-axis, $y$-axis, and $y=x$, we have $\hat{b}_{j}=\hat{a}_{2 j+1}=\hat{a}_{4 j+2}=0$ for $j=0,1,2, \cdots$. The truncated Taylor polynomial of $p(x$, $y$ ) (that is, $p(z)$ ) at some finite $4 n$ terms, where $n$ is a positive integer, is given by

$$
p_{4 n}(x, y)=\sum_{j=0}^{n} r^{4 j} \hat{a}_{4 j} \cos (4 j \theta) .
$$

Let $n=8$. By the result of [16], $p_{32}(x, y)$ is given by

$$
\begin{aligned}
p_{32}(x, y)= & 0.012057806957047935 \\
& -0.011754632105672258 r^{4} \cos (4 \theta) \\
& -0.00028938542181895446 r^{8} \cos (8 \theta) \\
& -0.000013152624399033289 r^{12} \cos (12 \theta) \\
& -0.0000006047527162152378 r^{16} \cos (16 \theta) \\
& -0.000000030379417891529316 r^{20} \cos (20 \theta) \\
& -0.0000000015825483904242497 r^{24} \cos (24 \theta) \\
& -0.00000000008646383804505168 r^{28} \cos (28 \theta) \\
& -0.000000000004011350923277267 r^{32} \cos (32 \theta) .
\end{aligned}
$$

By (4.6) and the above expression, an approximation of $G$ at $\tilde{z}=\xi+i \eta$ is given by

$$
\hat{G}(x, y ; \xi, \eta)=p_{32}(x, y)-\frac{1}{2 \pi} \ln (|x+i y-(\xi+i \eta)|) .
$$

Thus, approximated solutions of $U$ and $V$ of (4.3) and (4.4) are able to evaluate through an iterative scheme. A numerical method of finding an approximation of $a^{*}$ and $b^{*}$ is stated below.

Step 1: Assign a positive value for $a$. Choose a positive value for $b$ (say $b_{1}$ ). Set $\hat{U}_{0}(x, y)=0$ and $\hat{V}_{0}(x, y)=0$ for all $(x, y) \in \bar{D}$. Let $\hat{U}_{j}(x, y)$ and $\hat{V}_{j}(x, y)$ be approximated solutions of $U_{j}(x, y)$ and $V_{j}(x, y)$ given by

$$
\hat{U}_{j}(x, y)=\iint_{D} \frac{a}{1-\hat{V}_{j-1}(0,0)} \hat{G}(x, y ; \xi, \eta) \mathrm{d} \xi \mathrm{d} \eta,
$$




$$
\hat{V}_{j}(x, y)=\iint_{D} \frac{b}{1-\hat{U}_{j-1}(0,0)} \hat{G}(x, y ; \xi, \eta) \mathrm{d} \xi \mathrm{d} \eta .
$$

Compute $\hat{U}_{j}(x, y)$ and $\hat{V}_{j}(x, y)$ for $j=1,2,3, \cdots$ and $(x, y) \in D$. At this $b=b_{1}, \hat{U}_{j}$ and $\hat{V}_{j}$ are bounded by 1 and converge. That is, $\max _{\bar{D}} \hat{U}_{j}(x, y)<1$ and $\max _{\bar{D}} \hat{V}_{j}(x, y)<1$ for $j=1,2,3, \cdots$ and satisfy

$$
\begin{gathered}
\left|\max _{\bar{D}} \hat{U}_{j}(x, y)-\max _{\bar{D}} \hat{U}_{j-1}(x, y)\right|<1 \times 10^{-6}, \\
\left|\max _{\bar{D}} \hat{V}_{j}(x, y)-\max _{\bar{D}} \hat{V}_{j-1}(x, y)\right|<1 \times 10^{-6},
\end{gathered}
$$

for $j>N$ for some positive integer $N$.

Step 2: With the same value of $a$ in Step 1, choose another value for $b$ (say $b_{2}$ ). Set $\hat{U}_{0}(x, y)=0$ and $\hat{V}_{0}(x, y)=0$ for all $(x, y) \in \bar{D}$. To each $(x, y) \in D$. evaluate iterative integral (4.7) and (4.8) for $j=1,2,3, \cdots$. At this $b=b_{2}, \hat{U}_{j}$ and $\hat{V}_{j}$ do not exist. That is, $\max _{\bar{D}} \hat{U}_{j}(x, y) \geq 1$ and $\max _{\bar{D}} \hat{V}_{j}(x, y) \geq 1$ for some positive integer $j$. Calculate $b_{3}=\left(b_{1}+b_{2}\right) / 2$. Then, at $b=b_{3}$, evaluate (4.7) and (4.8) and compute $\max _{\bar{D}} \hat{U}_{j}$ and $\max _{\bar{D}} \hat{V}_{j}$ for $j=1,2,3, \cdots$.

Step 3: Set $b_{1}=b_{3}$ if $\max _{\bar{D}} \hat{U}_{j}(x, y)<1$ and $\max _{\bar{D}} \hat{V}_{j}(x, y)<1$ for $j=1,2,3, \cdots$, and satisfy

$$
\begin{aligned}
& \left|\max _{\bar{D}} \hat{U}_{j}(x, y)-\max _{\bar{D}} \hat{U}_{j-1}(x, y)\right|<1 \times 10^{-6}, \\
& \left|\max _{\bar{D}} \hat{V}_{j}(x, y)-\max _{\bar{D}} \hat{V}_{j-1}(x, y)\right|<1 \times 10^{-6},
\end{aligned}
$$

for $j>N$ for some positive integer $N$. Otherwise, set $b_{2}=b_{3}$ if $\max _{\bar{D}} \hat{U}_{j}(x, y) \geq 1$ and $\max _{\bar{D}} \hat{V}_{j}(x, y) \geq 1$ for some positive integer $j$. This procedure stops when $b_{1} \neq b_{3}$ and $\left|b_{1}-b_{3}\right|<1 \times 10^{-6}$ (or $\left|b_{2}-b_{3}\right|<1 \times 10^{-6}$ if $\left.b_{2} \neq b_{3}\right)$. Then, set $b^{*}=b_{3}$ and $a^{*}=a$. Otherwise, calculate $b_{3}=\left(b_{1}+b_{2}\right) / 2$. Then, at $b=b_{3}$, evaluate (4.7) and (4.8), and compute $\max _{\bar{D}} \hat{U}_{j}$ and $\max _{\bar{D}} \hat{V}_{j}$ for $j=1,2,3, \cdots$. Then, repeat Step 3 .

When we evaluate (4.7) and (4.8), the domain $D$ is divided into $225(15 \times 15)$ grid points uniformly. The B-Spline interpolation is used to interpolate the function value at these grid points. We use Mathematica to evaluate (4.7) and (4.8). As examples, we compute two groups of approximated values of $a^{*}$ and $b^{*}$. In the first group, we set $a=0.250000$ and vary the value of $b$. In the second one, we let $a=b$, then they change together. The results are listed in Table 1 .

\section{Conclusion}

In this paper, we prove that $u$ and $v$ reach their maximum at $(0,0)$ for all $t>0$. Lower and upper bounds of $u_{t}$ and $v_{t}$ at $(0,0)$ are obtained. From these results, we then show that $u$ and $v$ quench simultaneously at $(0,0)$. A numerical

Table 1. Approximated critical values.

\begin{tabular}{ccc}
\hline Group & Approximated $a^{*}$ & Approximated $b^{*}$ \\
\hline 1 & 0.250000 & 1.801318 \\
2 & 0.848362 & 0.848362 \\
\hline
\end{tabular}


method is introduced to compute approximated critical values of the semilinear parabolic system, and two sets of result are reported.

\section{Acknowledgements}

The author thanks the anonymous referees for their suggestions.

\section{Conflicts of Interest}

The author declares no conflicts of interest regarding the publication of this paper.

\section{References}

[1] Bimpong-Bota, K., Ortoleva, P. and Ross, J. (1974) Far-from-Equilibrium Phenomena at Local Sites of Reaction. The Journal of Chemical Physics, 60, 3124-3133. https://doi.org/10.1063/1.1681498

[2] Ortoleva, P. and Ross, J. (1972) Local Structures in Chemical Reactions with Heterogeneous Catalysis. The Journal of Chemical Physics, 56, 4397-4400. https://doi.org/10.1063/1.1677879

[3] Li, H. and Wang, M. (2004) Blow-Up Properties for Parabolic Systems with Localized Nonlinear Sources. Applied Mathematics Letters, 17, 771-778. https://doi.org/10.1016/j.aml.2004.06.004

[4] Chadam, J.M., Peirce, A. and Yin, H.M. (1992) The Blowup Property of Solutions to Some Diffusion Equations with Localized Nonlinear Reactions. Journal of Mathematical Analysis and Applications, 169, 313-328. https://doi.org/10.1016/0022-247X(92)90081-N

[5] Kawarada, H. (1975) On Solutions of Initial-Boundary Problem for $u_{t}=u_{x x}+1 /(1-u)$. Publications of the Research Institute for Mathematical Sciences, 10, 729-736.

[6] Nouaili, N. (2011) A Liouville Theorem for a Heat Equation and Applications for Quenching. Nonlinearlity, 24, 797-832. https://doi.org/10.1088/0951-7715/24/3/005

[7] Chang, C., Hsu, Y. and Liu, H.T. (2014) Quenching Behavior of Parabolic Problems with Localized Reaction Term. Mathematics and Statistics, 2, 48-53.

[8] Chan, C.Y. and Yang, J. (2001) No Quenching in Infinite Time for Degenerate Singular Semilinear Parabolic Equations. Applied Mathematics and Computation, 121, 29-35. https://doi.org/10.1016/S0096-3003(99)00276-3

[9] Evans, L.C. (1998) Partial Differential Equations. In: Graduate Studies in Mathematics, American Mathematical Society, Providence, RI, 502-504.

[10] Pao, C.V. (1992) Nonlinear Parabolic and Elliptic Equations. Plenum Press, New York, 54, 96, 532-533.

[11] Protter, M.H. and Weinberger, H.F. (1984) Maximum Principles in Differential Equations. Springer-Verlag, New York, 170. https://doi.org/10.1007/978-1-4612-5282-5

[12] Samarskii, A.A., Galaktionov, V.A., Kurdyumov, S.P. and Mikhailov, A.P. (1995) Blow-Up in Quasilinear Parabolic Equations. Walter de Gruyter, New York, 10-11. https://doi.org/10.1515/9783110889864

[13] Ladde, G.S., Lakshmikantham, V. and Vatsala, A.S. (1985) Monotone Iterative Techniques for Nonlinear Differential Equations. Pitman, Gloucester, NJ, 139.

[14] Chan, C.Y. (1993) Computation of the Critical Domain for Quenching in an Elliptic 
Plate. Neural, Parallel and Scientific Computations, 1, 153-162.

[15] Kythe, P.K. (2011) Green's Functions and Linear Differential Equations: Theory, Applications, and Computation. Chapman and Hall/CRC, Boca Raton, FL, 288, 304. https://doi.org/10.1201/b10494

[16] Chan, W.Y. (2016) Determining the Critical Domain of Quenching Problems for Coupled Nonlinear Parabolic Differential Equations. Proceedings of Dynamic Systems of Applications, 7, 63-69. 